\title{
Introduction to the Special Issue on Artificial Intelligence and Human-Robot Interaction
}

\author{
BRADLEY HAYES, University of Colorado Boulder, USA \\ MAYA CAKMAK, University of Washington, USA \\ STEPHANIE ROSENTHAL, Chatham University, USA
}

\begin{abstract}
ACM Reference format:
Bradley Hayes, Maya Cakmak, and Stephanie Rosenthal. 2018. Introduction to the Special Issue on Artificial Intelligence and Human-Robot Interaction. ACM Trans. Hum.-Robot Interact. 7, 2, Article 13 (October 2018), 3 pages.

https://doi.org/10.1145/3279995
\end{abstract}

Artificial Intelligence (AI) has had a transformational impact on Human-Robot Interaction (HRI) research over the past decade, enabling work in HRI to develop and investigate robots that can operate autonomously in far more challenging environments and far more complex scenarios than was possible ever before. Beyond laboratory studies, robots that explicitly interact with people as part of their functionality are increasingly being developed, productized, and deployed throughout the world, enabling ecologically valid ethnographic studies of interactions between humans and robots. These advances have been fueled by enabling technologies across many subfields of AI including machine learning, computer vision, task and motion planning, natural language understanding, and dialogue systems.

It is not, however, the case that AI research produced polished, ready-off-the-shelf tools that researchers could pick up and effortlessly use to build their envisioned autonomous robot. Rather, the shift has been due to a new, hybrid approach to human-centered robotics research, facilitated by HRI researchers who acquired deep technical skill sets and an influx of AI researchers applying their expertise to HRI problems. More interdiscplinary research teams consisting of formerly AI and HRI researchers also formed, resulting in a vibrant sub-community at the intersection of AI and HRI who came together at the AAAI Fall Symposium on AI for Human-Robot Interaction for the last 4 years. This special issue was encouraged by the continued success and overwhelming popularity of this symposium. Our goal is to exemplify this community's mature, high-quality, and original work, establishing T-HRI as a premier venue for work at the intersection of AI and HRI.

Research at this intersection is particularly challenging due to the very need for interdiscplinary, multi-faceted skill sets. AI-HRI researchers need to both innovate in computational techniques and

\footnotetext{
Authors' addresses: B. Hayes, University of Colorado Boulder, USA; email: Bradley.Hayes@colorado.edu; M. Cakmak, University of Washington, USA; email: mcakmak@cs.washington.edu; S. Rosenthal, Chatham University, USA; email: S.Rosenthal@chatham.edu.

Permission to make digital or hard copies of part or all of this work for personal or classroom use is granted without fee provided that copies are not made or distributed for profit or commercial advantage and that copies bear this notice and the full citation on the first page. Copyrights for third-party components of this work must be honored. For all other uses, contact the owner/author(s).

2018 Copyright is held by the owner/author(s).

2573-9522/2018/10-ART13

https://doi.org/10.1145/3279995
} 
rigorously study interactions. While AI researchers primarily work with datasets or well-defined problem specifications, HRI necessitates interactivity with a human in the loop. This makes collection of representative datasets or standardization of problems much more difficult. Furthermore, while AI research can compartmentalize different capabilities, such as vision or natural language understanding, and address them separately, studying human interactions with an autonomous robot requires a fully integrated system with at least minimal capabilities in all aspects of robotics. The four papers featured in this special issue are great examples of overcoming these challenges, contributing novel AI techniques as well as empirical understandings of how people interact with robots enabled by state-of-the-art AI capabilities.

In "Robot Classification of Human Interruptibility, and a Study of Its Effects" Banerjee et al. introduce a mobile robot that uses social and contextual cues to determine the best time to interrupt a person during a task. The authors first apply different temporal and non-temporal models for the interruptibility classification task and identify the features that enable most accurate prediction. The authors deployed their mobile robot for a large-scale user evaluation. They found that while people are resilient to interruptions in their tasks, robots with interruptibility-awareness were perceived as more social and improved their own task performance. These results underline the importance of testing the impact of intelligent systems in the wild.

In the domain of robot assistance "Learning and Personalizing Socially Assistive Robot Behaviors to Aid with Activities of Daily Living” by Moro et al. addresses the challenge of personalizing robot interactions to the unique needs of people with cognitive impairments. The authors propose a method that combines learning from demonstration, which allows caregivers to demonstrate assistive behaviors, with reinforcement learning, which refines the behavior to a personalized policy based on the user's cognition level. They implement this method on the socially assistive robot Casper, focusing on the task of making tea. The authors demonstrate that their method resulted in personalized policies, requiring less time from caregivers to teach the task.

Continuing on the theme of efficient robot learning from users, in "Human-Guided Object Mapping for Task Transfer" Fitzgerald et al. focus on enabling transfer learning through object mappings learned from human teachers. The authors propose a structured interaction with the teacher to infer an object mapping for task transfer. Through an interactive user study and offline assessment of task confidence, they demonstrate that human guidance increases the number of correct object mappings, while decreasing the effort required from humans to redemonstrate in the absence of transfer learning.

Finally, "Interaction Algorithm Effect on Human Experience" by Krening and Feigh tackles the design of interaction methods for learning algorithms to improve people's experiences with teaching, while simultaneously improving the resultant model accuracy. They studied two methods of natural language instruction: critique and action advice. The authors conducted an experiment to understand how different instruction types affected the same underlying learning algorithm. Their results show participants had better experiences in terms of frustration and perceived performance when they taught an agent using action advice compared to binary critique. They identify nine main characteristics of an interactive machine learning algorithm's design that impact the human's experience with teaching it. These results can potentially be applied to any intelligent agent.

An overarching goal of HRI research is to enable the development of capable autonomous robots that intelligently model, learn from, adapt to, and interact with humans. As such, the innovations occuring at the intersection of AI and HRI are central to its realization. With the continued, rapid pace of advancement within the fields of Artificial Intelligence and Robotics, there has never been a more exciting time to be part of this community, working to address core research challenges at the heart of both fields including those showcased within this special issue, as well as grand 
challenges at the intersections of social interaction, robotic planning and control, learning, and beyond. The guest editors hope that the articles featured in this special issue will inspire further research at the intersection of HRI and AI and foster productive interdisciplinary research findings. We wish to thank all of the contributors, reviewers, and editors without whom the realization of this special issue would not have been possible. 